\title{
Ruxolitinib for myelofibrosis
}

\author{
LIAN GU $^{1 *}$, LI SU ${ }^{2 *}$, QING CHEN ${ }^{1 *}$, JUANJUAN XIE ${ }^{1}$, GUANGLIANG WU ${ }^{1}$, \\ YAN YAN $^{1}$, BAOYUN LIANG $^{1}$, JINJING TAN $^{1}$ and NONG TANG ${ }^{1,3}$ \\ ${ }^{1}$ Department of Internal Neurology, First Affiliated Hospital, Guangxi University of Chinese Medicine; \\ ${ }^{2}$ School of Public Health of Guangxi Medical University; \\ ${ }^{3}$ Principal's Office, Guangxi University of Chinese Medicine, Nanning, Guangxi, P.R. China
}

Received November 1, 2012; Accepted December 24, 2012

DOI: $10.3892 / \mathrm{etm} .2013 .886$

\begin{abstract}
The aim of the present study was to assess the beneficial and harmful effects of ruxolitinib in patients with myelofibrosis (MF). The Cochrane databases, PubMed and Embase were searched for studies published up to October 2012. Randomised controlled trials assessing ruxolitinib versus a placebo or the best available therapy in patients with MF were included. Two trials randomised 528 patients with MF to ruxolitinib versus a placebo or ruxolitinib versus the best available therapy. Compared with the placebo, ruxolitinib had a significant beneficial effect on the proportion of patients that had a reduction in spleen volume of $\geq 35 \%$ at 24 weeks [odds ratio (OR), 109.78; 95\% confidence interval (CI), 14.97-804.78] or an increased overall survival rate (OR, 2.02; 95\% CI, 0.99-4.12). Ruxolitinib significantly increased the risk of several non-haematological or haematological adverse events, but not the risk of treatment discontinuations (OR, 1.04; 95\% CI, 0.50-2.14). Compared with the best available therapy, ruxolitinib had a significant beneficial effect on the proportion of patients that had a reduction in spleen volume of $\geq 35 \%$ at 24 (OR, $68.45 ; 95 \%$ CI, 4.15-1129.19) or 48 weeks (OR, 56.20; 95\%CI, 3.40-928.67). Ruxolitinib once again significantly increased the risk of several non-haematological adverse events, serious adverse events and dose reductions or interruptions (OR, 9.60; 95\% CI, 4.66-19.81), but not the risk of treatment discontinuations (OR, 1.54; 95\% CI, 0.48-4.97). In conclusion, based on the trials included in the present study, the use of ruxolitinib is beneficial in the treatment of MF.
\end{abstract}

Correspondence to: Dr Nong Tang, Guangxi University of Chinese Medicine, 179 Mingxiu East Road, Nanning, Guangxi 530001, P.R. China

E-mail: tangnong2012@yahoo.com.cn

Professor Lian Gu, First Affiliated Hospital, Guangxi University of Chinese Medicine, 89-9 Dongge Road, Nanning, Guangxi 530023, P.R. China

E-mail: gulian2012@163.com

*Contributed equally

Key words: ruxolitinib, myelofibrosis, randomised controlled trial

\section{Introduction}

Myelofibrosis (MF) is a rare disorder of the bone marrow which is characterised by excessive production of reticulin and collagen fibers (1). MF includes primary MF (PMF), post-essential thrombocythaemia (post-ET) MF and post-polycythaemia vera (post-PV) MF (2). Among the Philadelphia chromosome-negative chronic myeloproliferative neoplasms (MPNs), MF is the most symptomatic and carries the worst prognosis (3). According to epidemiological studies, it is estimated that the incidence of MF may be as high as 1.5 per 100,000 individuals. In clinical series, the median age at diagnosis for the majority of patients was $\sim 65$ years. In total, $\sim 22 \%$ of the cases occur in patients younger than 56 years old and $\sim 11 \%$ in patients younger than 46 years old (2). Other studies (4-8) indicate that $10-15 \%$ of cases may transform into secondary MF by the end of the second decade subsequent to PV or ET diagnosis. In 2010, MF continued to be the MPN that caused the highest morbidity and was corelated with the poorest life expectancy (9). The most common abnormalities in patients with MF include progressive splenomegaly, cytopenias and bothersome constitutional symptoms (e.g., unintentional weight loss, fevers and debilitating fatigue) (10). MF is also able to induce blastic transformation (11) and mortality (12).

Numerous conventional therapeutic modalities have been used in MF treatment as additional supportive treatments (13). Until recently, allogeneic haematopoietic stem cell transplantation has been the only potentially curative treatment for patients with MF, but an option that was traditionally only feasible for a small percentage of patients, namely the younger and physically fit. However, recent studies suggest that it may be utilised in older individuals as well $(14,15)$. Other therapeutic modalities (e.g., thalidomide, hydroxyurea, corticosteroids, anagrelide, androgens, splenectomy or spleen irradiation, transfusions, pirfenidone and suramin) are only palliative and do not provide a substantial improvement in survival rates (16-32).

Ruxolitinib (formerly known as INC424 or INCB18424) is an orally bioavailable, selective Janus kinase (JAK) 1 and 2 inhibitor approved by the US Food and Drug Administration for the treatment of MF. The mechanism of action of ruxolitinib is the attenuation of cytokine signalling by the inhibition of JAK1 and JAK2 (wild-type or mutated forms), leading to 
antiproliferative and proapoptotic effects. On the basis of the results of a phase II clinical trial in patients with MF, ruxolitinib showed durable efficacy in the reduction of splenomegaly and circulating pro-inflammatory cytokines. Ruxolitinib recipients also exhibited improvements in physical activity, weight gain, existing symptoms (including constitutional symptoms) and parameters gauging quality of life. These findings were validated by two phase III clinical MF studies $(1,33,34)$.

Conventional medications for treating MF are largely palliative and rarely provide durable clinical benefits, whereas stem cell transplantation appears to be restricted to a minority of patients. Given the limitations already discussed, more effective disease-targeted therapeutic approaches are required for patients with MF. Based on the efficacy and tolerability reported in completed clinical trials, ruxolitinib now has an important place among currently available treatment modalities. In the present systematic review, the beneficial and harmful effects of ruxolitinib in patients with MF are assessed.

\section{Materials and methods}

Data sources. The Cochrane Central Register of Controlled Trials in The Cochrane Library, PubMed and Embase were searched for studies published up to October 2012 using the search terms 'myelofibrosis', 'ruxolitinib' and 'INCB-018424'.

Study selection. Randomised controlled trials assessing ruxolitinib versus no intervention, a placebo or the best available therapy in patients with intermediate risk, high risk or not determined MF, including PMF, post-PV MF and post-ET MF, were included, irrespective of gender, age or ethnic origin.

Outcome measures. The primary outcome measures were: i) the proportion of patients that had a reduction in spleen volume of $\geq 35 \%$ at 24 weeks; ii) the proportion of patients that had a reduction in spleen volume of $\geq 35 \%$ at 48 weeks. The secondary outcome measures were: i) overall survival rate; ii) all adverse events, including non-haematological and haematological adverse events, serious adverse events, necessary dose reductions or interruptions and treatment discontinuations.

Assessment of trial methodological quality. The methodological quality of each trial included in the review was assessed by two authors using the quality criteria specified in the Cochrane Handbook for Systematic Reviews of Interventions 5.1.0 (35). The assessment was based on adequate sequence generation, allocation concealment, blinding, selective outcome reporting and other sources of bias. Any disagreements were resolved through consensus. Each criteria was classified as 'yes', 'no' or 'unclear'. A classification of 'yes' indicated the above assessment methods had been used and were specifically described, 'no' if it did not mention the above assessment methods, and 'unclear' if it only mentioned the above assessment methods had been used but did not specifically describe them. The summary assessments for the bias risk for each significant outcome within and across studies were classified as 'low risk of bias', 'high risk of bias' and 'unclear risk of bias'.

Data extraction. Data were extracted independently by two authors in a standard format. Any disagreements were resolved through consensus. Authors of the eligible studies were contacted to request missing data that were required for further analysis. The primary author, country, study design, sample size, age range, number of males and females, initial dose, intervention, median follow-up, methodological quality of the trial and the outcome measures were extracted from each trial as described.

Data synthesis. Data synthesis and analysis was performed following the recommendations of Cochrane Review Manager software, RevMan version 5.1.0. All data were analysed on the basis of an intention to treat and therefore included all patients irrespective of compliance or follow-up. The random-effects (36) and fixed-effects models (37) were used for analysing the data. If the models provided the same result with regard to statistical significance, only the results of the fixed-effect model were presented. Odds ratios (ORs) with 95\% confidence intervals (CIs) were used for the analysis of all outcomes. Heterogeneity was explored by the Chi-square test, with the significance set at a P-value of 0.10 and measured by $\mathrm{I}^{2}$ (38). Possible sources of heterogeneity were assessed by sensitivity and subgroup analyses as described later. Funnel plots and other analytical methods were used to assess potential bias, depending on the number of clinical trials included (39).

Subgroup analyses and sensitivity analysis. In order to explore the effect of size differences, the present study aimed to perform analyses on the following subgroups: i) MF type (PMF, post-PV MF or post-ET MF); ii) IPSS risk group (intermediate-2, high or not determined ); iii) age ( $\leq 65$ or $>65$ years); iv) JAK2V617F mutation status (presence or absence); v) baseline palpable spleen length $(\leq 10$ or $>10 \mathrm{~cm})$; vi) baseline haemoglobin level ( $\geq 10$ or $<10 \mathrm{~g} / \mathrm{dl}$ ); vii) gender. Sensitivity analyses were performed to exclude the trials which would potentially bias the results.

\section{Results}

Search results. A total of 307 references were identified through electronic searches of the Cochrane Central Register of Controlled Trials in the Cochrane Library $(n=2)$, PubMed $(n=8)$ and Embase $(n=223)$. Duplicates, non-clinical studies, clearly irrelevant articles or studies with a different purpose $(n=303)$ were excluded. There were no quasi-randomised studies identified. A total of 4 references were retrieved for further assessment and another 2 non-randomised control trials were excluded. All included trials had been published as full manuscripts.

Characteristics of included studies. Together the two trials included 528 patients and used a parallel group design. The first trial, conducted by Verstovsek et al (33), used a placebo control, while the second trial, conducted by Harrison et al (34), used the best available therapy as a control. The first trial was conducted at 89 sites in the United States, Australia and Canada and patients were randomised (1:1) to receive ruxolitinib or a placebo. Patients of the second trial were randomised (2:1) to ruxolitinib or to the best available therapy. The mean age (range, 35-91 years) of the patients included and the proportion of males (55.3\%) was reported in each trial. The 


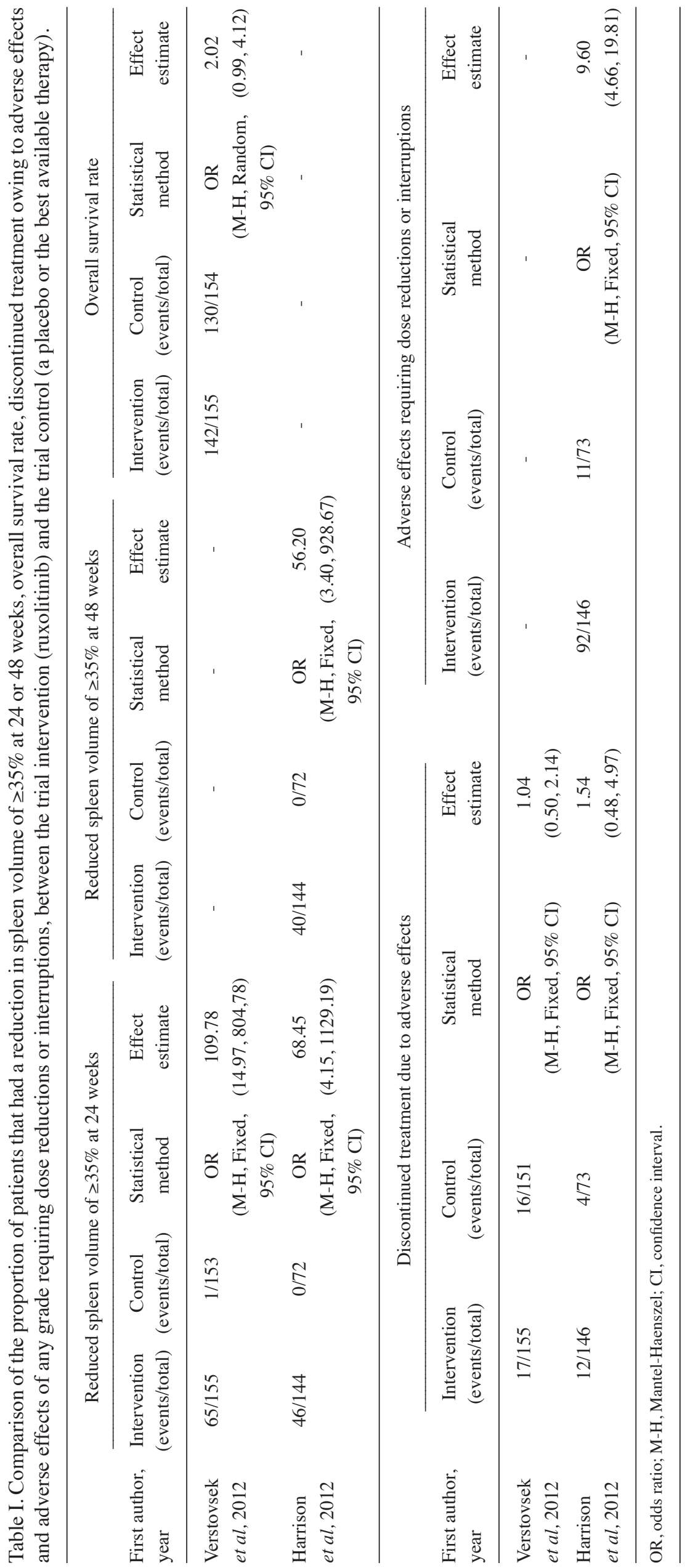


median spleen volume was $>2,300 \mathrm{~cm}^{3}(>10$ times the median normal spleen volume of $200 \mathrm{~cm}^{3}$ ). A total of $38.8 \%$ of the patients had an IPSS intermediate-2-risk disease, $60.61 \%$ had a high-risk disease and $0.1 \%$ were not determined. A total of $51.1 \%$ of the patients were categorized as PMF, $31.3 \%$ as post PV MF and $17.6 \%$ as post ET MF. The proportion of patients who underwent previous MF therapy with hydroxyurea and radiotherapy was 66.48 and $1.82 \%$, respectively. On the basis of the baseline peripheral blood platelet count (Plt), in the first trial (33) the initial dose of ruxolitinib was $15 \mathrm{mg} / \mathrm{bid}$ (Plt, $\left.100-200 \times 10^{9} / \mathrm{l}\right)$ or $20 \mathrm{mg} / \mathrm{bid}\left(\mathrm{Plt},>200 \times 10^{9} / \mathrm{l}\right)$. In the second trial (34) the initial dose of ruxolitinib was also 15 or $20 \mathrm{mg} /$ bid and was adjusted to within the range of 5 to $25 \mathrm{mg} / \mathrm{bid}$.

Risk of bias in included studies. Each study only described a randomisation but did not describe the method used for generating and concealing the allocations, so were consequently assessed as 'unclear'. The first trial (33) was described as double-blind and placebo-controlled. Although there was no information with regard to which aspects involved were blinded, it was assessed as 'yes'. The second trial (34) had no information on blinding, therefore it was uncertain whether blinding was used or not and consequently an assessment of 'unclear' was made. An intention-to-treat analysis was used in the two trials and each were assessed as 'yes'. All outcomes of the trials listed in their methods sections were reported in their results, therefore this criteria was also assessed as 'yes'. The first trial (33) was supported by Incyte and the second trial (34) was supported by Novartis pharmaceuticals, so each trial was assessed as 'no'.

Effects of interventions. It was not possible to perform meta-analysis as the two studies included had different comparison interventions and were not groupable.

Ruxolitinib versus placebo. In the trial conducted by Verstovsek et al (33), when compared with the placebo, ruxolitinib significantly increased the proportion of patients that had a reduction in spleen volume of $\geq 35 \%$ at 24 weeks (OR, 109.7; 95\% CI, 14.97-804.78). Ruxolitinib also had a significant beneficial effect on overall survival rate (OR, 2.02; 95\% CI, $0.99-4.12)$. There were no significant differences identified in the treatment discontinuations between ruxolitinib and the placebo (OR, 1.04; 95\% CI, 0.50-2.14). The most frequently reported grade 1 or 2 non-haematological and haematological adverse effects in the ruxolitinib group were fatigue and anaemia, respectively. The most frequently reported grade 3 or 4 non-haematological adverse effects in the ruxolitinib group were fatigue, abdominal pain, diarrhoea and arthralgia, while anaemia and thrombocytopenia were the most common haematological adverse effects. No data were reported on the proportion of patients that had a reduction in spleen volume of $\geq 35 \%$ at 48 weeks, serious adverse effects or adverse effects of any grade requiring dose reductions or interruptions (Table I).

Ruxolitinib versus bestavailable therapy. In the trial conducted by Harrison et al (34), when compared with the best available therapy, ruxolitinib significantly increased the proportion of patients that had a reduction in spleen volume of $\geq 35 \%$ at 24 weeks (OR, 68.45; 95\% CI, 4.15-1129.19). Ruxolitinib also significantly increased the proportion of patients that had a reduction in spleen volume of $\geq 35 \%$ at 48 weeks (OR, 56.20; 95\% CI, 3.40-928.67). There were no significant differences in the treatment discontinuations between ruxolitinib and the best available therapy (OR, 1.54; 95\% CI, 0.48-4.97), however ruxolitinib significantly increased all grades of adverse effects that required dose reductions or interruptions (OR, 9.60; 95\% CI, 4.66-19.81). In the ruxolitinib group, the most common serious adverse effect was anaemia. Other commonly reported serious adverse events included abdominal pain, pyrexia, esophageal varices, dyspnea and pneumonia. The non-haematological adverse event that occurred in any grade more frequently in the ruxolitinib group was diarrhoea [with diarrhoea of any grade occurring in 34 of 146 patients (23\%) and grade 3 or 4 diarrhoea occurring in 2 of 146 patients (1\%)]. The most common grade 3 or 4 non-haematological adverse event occurring in the ruxolitinib group was abdominal pain (occurring in $3 \%$ of the patients). Other commonly reported grade 3 or 4 non-haematological adverse events included abdominal pain, back pain and pyrexia. No data were reported on overall survival rates (Table I).

Subgroup and sensitivity analyses. Subgroup or sensitivity analyses were not conducted in the present study due to the lack of sufficient trial numbers.

\section{Discussion}

In total, the two trials randomised 528 patients with MF to ruxolitinib versus a placebo or ruxolitinib versus the best available therapy. Compared with the placebo, the present study identified that ruxolitinib had a significant beneficial effect on the proportion of patients that had a reduction in spleen volume of $\geq 35 \%$ at 24 weeks and an increased overall survival rate. Ruxolitinib significantly increased the risk of several non-haematological or haematological adverse events, but not the risk of treatment discontinuations. Compared with the best available therapy, ruxolitinib had a significant beneficial effect on the proportion of patients that had a reduction in spleen volume of $\geq 35 \%$ at 24 or 48 weeks. Ruxolitinib significantly increased the risk of several non-haematological adverse events, serious adverse events and dose reductions or interruptions, but not the risk of treatment discontinuations.

In the two randomised controlled trials on ruxolitinib in MF, the method to generate randomisation sequences was not stated, concealment of randomisation was not mentioned, information on blinding was not available in one study, the total number of included patients was small, follow-up was insufficient and no data were obtained on changes in marrow fibrosis or the JAK2V617F allele burden. A potential bias existed due to the limitations of the search strategy in the present study. Owing to insufficient experience among the authors, a second potential bias involved the assessment of the methodological quality of the trials included.

Based on the two trials, there is evidence to support the use of ruxolitinib for the treatment of MF. However, ruxolitinib should be used under close supervision from a physician as it is associated with the risks of potentially serious adverse effects, including anaemia and pyrexia. High-quality randomised controlled trials with longer follow-ups are required to reach 
firm conclusions with regard to the ability of ruxolitinib to modify the natural disease course.

\section{Acknowledgements}

This study was supported by grants from the National Natural Science Foundation of China (No. 81260594), the Significant Scientific Research Foundation of the Guangxi Health Department (Nos. 2012047 and 200933), the Significant Scientific Research Foundation of Guangxi University of Chinese Medicine (No. ZD2012007); and the Science and Technology Project of Traditional Chinese Medicine, Guangxi (Nos. GZKZ1107 and 200911LX203).

\section{References}

1. Ostojic A, Vrhovac R and Verstovsek S: Ruxolitinib for the treatment of myelofibrosis: its clinical potential. Ther Clin Risk Manag 8: 95-103, 2012.

2. Tefferi A: Myelofibrosis with myeloid metaplasia. N Engl J Med 342: 1255-1265, 2000

3. Vannucchi AM: Management of myelofibrosis. Hematology Am Soc Hematol Educ Program 2011: 222-230, 2011.

4. Alvarez-Larrán A, Cervantes F, Bellosillo B, et al: Essential thrombocythemia in young individuals: frequency and risk factors for vascular events and evolution to myelofibrosis in 126 patients. Leukemia 21: 1218-1223, 2007.

5. Najean Y, Rain JD, Dresch C, et al: Risk of leukaemia, carcinoma, and myelofibrosis in ${ }^{32} \mathrm{P}$ - or chemotherapy-treated patients with polycythaemia vera: a prospective analysis of 682 cases. The 'French Cooperative Group for the Study of Polycythaemias'. Leuk Lymphoma 22 (Suppl 1): 111-119, 1996.

6. Passamonti F, Rumi E, Arcaini L, et al: Prognostic factors for thrombosis, myelofibrosis, and leukemia in essential thrombocythemia: a study of 605 patients. Haematologica 93: 1645-1651, 2008.

7. Randi ML, Barbone E, Fabris F, Varotto L, Macri C and Girolami A: Post-polycythemia myeloid metaplasia: experience with a large cohort of patients. J Med 25: 363-369, 1994.

8. Wolanskyj AP, Schwager SM, McClure RF, Larson DR and Tefferi A: Essential thrombocythemia beyond the first decade: life expectancy, long-term complication rates, and prognostic factors. Mayo Clin Proc 81: 159-166, 2006.

9. Vardiman JW, Thiele J, Arber DA, et al: The 2008 revision of the World Health Organization (WHO) classification of myeloid neoplasms and acute leukemia: rationale and important changes. Blood 114: 937-951, 2009.

10. Mesa RA, Niblack J, Wadleigh M, et al: The burden of fatigue and quality of life in myeloproliferative disorders (MPDs): an international Internet-based survey of 1179 MPD patients Cancer 109: 68-76, 2007.

11. Mesa RA, Li CY, Ketterling RP, Schroeder GS, Knudson RA and Tefferi A: Leukemic transformation in myelofibrosis with myeloid metaplasia: a single-institution experience with 91 cases. Blood 105: 973-977, 2005.

12. Cervantes F, Dupriez B, Pereira A, et al: New prognostic scoring system for primary myelofibrosis based on a study of the International Working Group for Myelofibrosis Research and Treatment. Blood 113: 2895-2901, 2009.

13. Hennessy BT, Thomas DA, Giles FJ, Kantarjian H and Verstovsek S: New approaches in the treatment of myelofibrosis. Cancer 103: 32-43, 2005.

14. Barbui T, Barosi G, Birgegard G, et al: Philadelphia-negative classical myeloproliferative neoplasms: critical concepts and management recommendations from European LeukemiaNet. J Clin Oncol 29: 761-770, 2011.

15. Samuelson S, Sandmaier BM, Heslop HE, et al: Allogeneic haematopoietic cell transplantation for myelofibrosis in 30 patients 60-78 years of age. Br J Haematol 153: 76-82, 2011.
16. Löfvenberg E and Wahlin A: Management of polycythaemia vera, essential thrombocythaemia and myelofibrosis with hydroxyurea. Eur J Haematol 41: 375-381, 1988.

17. Martínez-Trillos A, Gaya A, Maffioli M, et al: Efficacy and tolerability of hydroxyurea in the treatment of the hyperproliferative manifestations of myelofibrosis: results in 40 patients. Ann Hematol 89: 1233-1237, 2010.

18. Steurer M, Gastl G, Jedrzejczak WW, et al: Anagrelide for thrombocytosis in myeloproliferative disorders: a prospective study to assess efficacy and adverse event profile. Cancer 101: 2239-2246, 2004.

19. Birgegård G, Björkholm M, Kutti J, et al: Adverse effects and benefits of two years of anagrelide treatment for thrombocythemia in chronic myeloproliferative disorders. Haematologica 89: 520-527, 2004

20. Sirhan S, Lasho TL, Hanson CA, Mesa RA, Pardanani A and Tefferi A: The presence of JAK2V617F in primary myelofibrosis or its allele burden in polycythemia vera predicts chemosensitivity to hydroxyurea. Am J Hematol 83: 363-365, 2008.

21. Mesa RA, Nagorney DS, Schwager S, Allred J and Tefferi A: Palliative goals, patient selection, and perioperative platelet management: outcomes and lessons from 3 decades of splenectomy for myelofibrosis with myeloid metaplasia at the Mayo Clinic. Cancer 107: 361-370, 2006.

22. Elliott MA, Chen MG, Silverstein MN and Tefferi A: Splenic irradiation for symptomatic splenomegaly associated with myelofibrosis with myeloid metaplasia. Br J Haematol 103: 505-511, 1998.

23. Besa EC, Nowell PC, Geller NL and Gardner FH: Analysis of the androgen response of 23 patients with agnogenic myeloid metaplasia: the value of chromosomal studies in predicting response and survival. Cancer 49: 308-313, 1982.

24. Shimoda K, Shide K, Kamezaki K, et al: The effect of anabolic steroids on anemia in myelofibrosis with myeloid metaplasia: retrospective analysis of 39 patients in Japan. Int J Hematol 85: 338-343, 2007.

25. Cervantes F, Alvarez-Larrán A, Domingo A, Arellano-Rodrigo E and Montserrat E: Efficacy and tolerability of danazol as a treatment for the anaemia of myelofibrosis with myeloid metaplasia: long-term results in 30 patients. Br J Haematol 129: 771-775, 2005.

26. Merup M, Kutti J, Birgergård G, et al: Negligible clinical effects of thalidomide in patients with myelofibrosis with myeloid metaplasia. Med Oncol 19: 79-86, 2002.

27. Barosi G, Elliott M, Canepa L, et al: Thalidomide in myelofibrosis with myeloid metaplasia: a pooled-analysis of individual patient data from five studies. Leuk Lymphoma 43: 2301-2307, 2002.

28. Mesa RA, Elliott MA, Schroeder G and Tefferi A: Durable responses to thalidomide-based drug therapy for myelofibrosis with myeloid metaplasia. Mayo Clin Proc 79: 883-889, 2004.

29. Abgrall JF, Guibaud I, Bastie JN, et al: Thalidomide versus placebo in myeloid metaplasia with myelofibrosis: a prospective, randomized, double-blind, multicenter study. Haematologica 91: 1027-1032, 2006

30. Thomas DA, Giles FJ, Albitar M, et al: Thalidomide therapy for myelofibrosis with myeloid metaplasia. Cancer 106: 1974-1984, 2006.

31. Mesa RA, Steensma DP, Pardanani A, et al: A phase 2 trial of combination low-dose thalidomide and prednisone for the treatment of myelofibrosis with myeloid metaplasia. Blood 101: 2534-2541, 2003.

32. Jabbour E, Thomas D, Kantarjian H, et al: Comparison of thalidomide and lenalidomide as therapy for myelofibrosis. Blood 118: 899-902, 2011

33. Verstovsek S, Mesa RA, Gotlib J, et al: A double-blind, placebocontrolled trial of ruxolitinib for myelofibrosis. N Engl J Med 366: 799-807, izdddds. Stat Med 6: 341-350, 1987.

34. Higgins JP, Thompson SG, Deeks JJ and Altman DG: Measuring inconsistency in meta-analyses. BMJ 327: 557-560, 2003.

35. Egger M, Davey Smith G, Schneider M and Minder C: Bias in meta-analysis detected by a simple, graphical test. BMJ 315 : 629-634, 1997. 